\title{
ON THE TRANSFORM OF A SINGULAR OR AN ABSOLUTELY CONTINUOUS MEASURE
}

\section{RAOUF DOSS}

Let $G$ be a locally compact abelian group with dual $\Gamma$. A wellknown theorem of Bochner [1], generalized by Eberlein [2], is the following:

"A continuous function $\varphi$ defined on $\Gamma$ is the Fourier-Stieltjes transform of a finite (regular) measure on $G$ if and only if there is a constant $A$ such that for every trigonometric polynomial

$$
p(x)=\sum c_{i}\left(-x, \gamma_{i}\right), \quad \gamma_{i} \in \Gamma,
$$

the relation $\|p\|_{\infty} \leqq 1$ implies $\left|\sum c_{i} \varphi\left(\gamma_{i}\right)\right| \leqq A . "$

If we take $A=\sup \left|\sum c_{i} \varphi\left(\gamma_{i}\right)\right|$ where the sup is over all polynomials $p$ for which $\|p\|_{\infty} \leqq 1$, then whatever be $\epsilon>0$, there is a polynomial

$$
p(x)=\sum c_{i}\left(-x, \gamma_{i}\right), \quad \gamma_{i} \in \Gamma,
$$

such that $\|p\|_{\infty} \leqq 1$ and $\left|\sum c_{i} \varphi\left(\gamma_{i}\right)\right|>A-\epsilon$.

A splitting of the last property gives very simple (mutually exclusive) characterizations of the transform of a singular or an absolutely continuous measure. We prove the following theorems:

Theorem 1. A continuous function $\varphi$ defined on $\Gamma$ is the FourierStieltjes transform of a singular measure on $G$ if and only if there is a constant $A$ such that

(i) for every trigonometric polynomial $p(x)=\sum c_{i}\left(-x, \gamma_{i}\right), \gamma_{i} \in \Gamma$, the relation $\|p\|_{\infty} \leqq 1$ implies $\left|\sum c_{i} \varphi\left(\gamma_{i}\right)\right| \leqq A$;

(iis) whatever be $\epsilon>0$ and the compact set $K$ in $\Gamma$ there is a polynomial

$$
p(x)=\sum c_{i}\left(-x, \gamma_{i}\right), \quad \gamma_{i} \in \Gamma, \gamma_{i} \notin K,
$$

such that $\|p\|_{\infty} \leqq 1$ and $\left|\sum c_{i} \varphi\left(\gamma_{i}\right)\right|>A-\epsilon$.

THEOREM 2. A continuous function $\varphi$ defined on $\Gamma$ is the transform of an absolutely continuous measure on $G$ if and only if

(i) there is a constant $A$ such that for every polynomial

$$
p(x)=\sum c_{i}\left(-x, \gamma_{i}\right), \quad \gamma_{i} \in \Gamma,
$$

the relation $\|p\|_{\infty} \leqq 1$ implies $\left|\sum c_{i} \varphi\left(\gamma_{i}\right)\right| \leqq A$;

Received by the editors January 1, 1967. 
(iia) whatever be $\epsilon>0$ there is a compact set $K$ in $\Gamma$ such that for every polynomial $p(x)=\sum c_{i}\left(-x, \gamma_{i}\right), \gamma_{i} \in \Gamma, \gamma_{i} \notin K$, the relation $\|p\|_{\infty} \leqq 1$ implies $\left|\sum c_{i} \varphi\left(\gamma_{i}\right)\right|<\epsilon$.

Proof of Theorem 1.

Necessity of (iis). Let $\varphi(\gamma)=\hat{\mu}_{s}(\gamma)$ where $\mu_{s}$ is singular and $\left\|\mu_{s}\right\|=A$. Let $\epsilon>0$ and the compact set $K$ in $\Gamma$ be given. There is a polynomial

$$
r(x)=\sum_{j=1}^{N} b_{j}\left(-x, \gamma_{j}^{\prime}\right), \quad \gamma_{j}^{\prime} \in \Gamma,
$$

such that $\|r\|_{\infty} \leqq 1$ and $\left|\sum b_{j} \hat{\mu}_{s}\left(\gamma_{j}^{\prime}\right)\right|=\left|\int_{G} r(x) d \mu_{8}(x)\right|>A-\epsilon$.

The set $C=\left\{-\gamma_{1}^{\prime}, \cdots,-\gamma_{N}^{\prime}\right\} \cup-K$ being compact, there is a $k \in L^{1}(G)$ such that $\hat{k}(\gamma)=1$ on $C$; i.e., $\hat{k}\left(-\gamma_{j}^{\prime}\right)=1, j=1, \cdots, N$, and $\hat{k}(-\gamma)=1$ for $\gamma \in K$ and such that $\|k\|_{1}<1+\epsilon$ (see, e.g., $[3$, p. 53]). Put $k^{\prime}(x)=k(-x)$ and $f(x)=\left(k^{\prime} * \mu_{s}\right)(x)$. Then $f \in L^{1}(G)$ and $\hat{f}(\gamma)=\hat{k}(-\gamma) \hat{\mu}_{s}(\gamma)$ for $\gamma \in \Gamma$. Also

$$
\begin{aligned}
\left|\int_{G} r(x) f(x) d x\right| & =\left|\sum b_{j} \hat{f}\left(\gamma_{j}^{\prime}\right)\right|=\mid \sum b_{j} \hat{k}\left(-\gamma_{j}^{\prime}\right) \hat{\mu}_{s}\left(\gamma_{j}^{\prime}\right) । \\
& =\left|\sum b_{j} \hat{\mu}_{s}\left(\gamma_{j}^{\prime}\right)\right|>A-\epsilon .
\end{aligned}
$$

Hence $\|f\|_{1}>A-\epsilon$.

Now consider the measure $d \mu=d \mu_{s}-f(x) d x$. We have $\|\mu\|$ $=\left\|\mu_{s}\right\|+\|f\|_{1}>2 A-\epsilon$. Hence there exists a polynomial $q(x)$ $=\sum d_{i}\left(-x, \gamma_{i}\right)$ such that $\|q\|_{\infty} \leqq 1$ and $\left|\sum d_{i} \hat{\mu}\left(\gamma_{i}\right)\right|>2 A-\epsilon$. Put $p(x)=\frac{1}{2}[q(x)-(q * k)(x)]$. Then

$$
p(x)=\sum c_{i}\left(-x, \gamma_{i}\right)
$$

where $c_{i}=\frac{1}{2}\left[d_{i}-d_{i} \hat{k}\left(-\gamma_{i}\right)\right]$ so that

$$
c_{i}=0 \quad \text { for } \gamma_{i} \in K \text {. }
$$

Also,

$$
\|p\|_{\infty} \leqq \frac{1}{2}+\frac{1}{2}(1+\epsilon)=1+\epsilon / 2
$$

Now

$$
\begin{aligned}
\sum c_{i} \hat{\mu}_{s}\left(\gamma_{i}\right) & =\frac{1}{2} \sum\left[d_{i} \hat{\mu}_{s}\left(\gamma_{i}\right)-d_{i} \hat{\mu}_{s}\left(\gamma_{i}\right) \hat{k}\left(-\gamma_{i}\right)\right] \\
& =\frac{1}{2} \sum d_{i}\left[\hat{\mu}_{s}\left(\gamma_{i}\right)-\hat{f}\left(\gamma_{i}\right)\right]=\frac{1}{2} \sum d_{i} \hat{\mu}\left(\gamma_{i}\right) .
\end{aligned}
$$

Hence

$$
\left|\sum c_{i} \hat{\mu}_{s}\left(\gamma_{i}\right)\right|>A-\epsilon / 2 .
$$

Since $\epsilon>0$ is arbitrary, relations (1) and (2), (3) and (4) prove the necessity of (iis). 
Sufficiency of (i) and (iis). We know already by Bochner's theorem that $\varphi$ is the transform of a regular finite measure $\mu$, where $\|\mu\|=A$. We show that $\mu$ is singular. Let $d \mu=d \mu_{s}+g(x) d x$ be the Lebesgue decomposition of $\mu$. $\epsilon>0$ being given, there is an $h \in L^{1}(G)$, whose transform $\hat{h}$ vanishes outside some compact set $K$, and such that $\|g-h\|_{1}<\epsilon$. Put $d v=d \mu-h(x) d x=d \mu_{s}+(g-h) d x$. Then $\hat{v}(\gamma)$ coincides with $\varphi(\gamma)$ outside $K$. Put $A_{s}=\left\|\mu_{s}\right\|$. By (iis) there is a polynomial

$$
p(x)=\sum c_{i}\left(-x, \gamma_{i}\right), \quad \gamma_{i} \notin K,
$$

such that $\|p\|_{\infty} \leqq 1$ and $\left|\sum c_{i}\left(\hat{g}\left(\gamma_{i}\right)-\hat{h}\left(\gamma_{i}\right)\right)+\sum c_{i} \hat{\mu}_{s}\left(\gamma_{i}\right)\right|>A-\epsilon$. The 1.h.s. is at most $A_{s}+\epsilon$. Therefore, $A_{s}+\epsilon \geqq A-\epsilon$, i.e., $A_{s} \geqq A-2 \epsilon$. Since $A_{s} \leqq A$ we conclude $A_{s}=A$ and therefore $\|g\|_{1}=0$. Thus $\mu$ is singular and the proof is complete.

Proof of TheOREM 2.

Necessity of (iia). Let $\varphi$ be the transform $\hat{f}$ of some $f \in L^{1}(G)$ and let $\epsilon>0$ be given. There is a $k \in L^{1}(G)$ with compact support $K$, such that $\|f-f * k\|_{1}<\epsilon$. If now $p(x)=\sum c_{i}\left(-x, \gamma_{i}\right), \gamma_{i} \in \Gamma, \gamma_{i} \notin K$ with $\|p\|_{\infty} \leqq 1$, then, for $p^{\prime}(x)=p(-x)$,

$$
\begin{aligned}
\left|\sum c_{i} \varphi\left(\gamma_{i}\right)\right| & =\left|\sum c_{i} \hat{f}\left(\gamma_{i}\right)\right|=\left|\sum c_{i}\left(\hat{f}\left(\gamma_{i}\right)-\hat{k}\left(\gamma_{i}\right) \hat{f}\left(\gamma_{i}\right)\right)\right| \\
& =\left|\left(p^{\prime} *(f-k * f)\right)(0)\right| \leqq\left\|p^{\prime}\right\|_{\infty} \cdot\|f-k * f\|_{1}<\epsilon .
\end{aligned}
$$

This proves the necessity of (iia).

Sufficiency of (i) and (iia). We know already that $\varphi$ is the transform $\hat{\mu}$ of some finite measure on $G$. Let $d \mu=d \mu_{8}+f(x) d x$ be the Lebesgue decomposition of $\mu$. Put $A_{s}=\left\|\mu_{s}\right\|$. Let $\epsilon>0$ be given. Let $K_{1}$ be the compact set in $\Gamma$ associated to $\epsilon$ by (iia), and let $K_{2}$ be the compact set associated to $\epsilon$ and the absolutely continuous measure $f(x) d x$ by the necessity just proved. Put $K=K_{1} \cup K_{2}$. By Theorem 1 there is a polynomial $p(x)=\sum c_{i}\left(-x, \gamma_{i}\right), \gamma_{i} \in \Gamma, \gamma_{i} \notin K$, such that $\|p\|_{\infty} \leqq 1$ and $\left|\sum c_{i} \hat{\mu}_{s}\left(\gamma_{i}\right)\right|>A_{s}-\epsilon$. Also, $\left|\sum c_{i} \varphi\left(\gamma_{i}\right)\right|<\epsilon$ since $K_{1} \subset K$ and $\left|\sum c_{i} \hat{f}\left(\gamma_{i}\right)\right|<\epsilon$ since $K_{2} \subset K$. We conclude

$$
A_{s}-\epsilon<\left|\sum c_{i} \hat{\mu}_{s}\left(\gamma_{i}\right)\right|=\left|\sum c_{i}\left(\varphi\left(\gamma_{i}\right)-\hat{f}\left(\gamma_{i}\right)\right)\right|<2 \epsilon,
$$

i.e., $A_{s}<3 \epsilon$. Since $\epsilon$ is arbitrary, we conclude $A_{s}=0$ so that $d \mu_{s}=0$ and $\mu$ is absolutely continuous. This completes the proof of the theorem.

\section{REFERENCES}

1. S. Bochner, $A$ theorem on Fourier-Stieltjes integrals, Bull. Amer. Math. Soc. 40 (1934), 271-276.

2. W. F. Eberlein, Characterizations of Fourier-Stieltjes transforms, Duke Math. J. 22 (1955), 465-468.

3. W. Rudin, Fourier analysis on groups, Interscience, New York, 1962.

State University of New York, Stony Brook 